\title{
Usefulness of the delta neutrophil index to predict 30-day mortality in patients with ST segment elevation myocardial infarction
}

Taeyoung Kong, $M D^{1}$, Tae Hoon Kim, $M D^{2}$, Sung Phil Chung, $M D^{1}$, Je Sung You, $M D^{1}$

1. Department of Emergency Medicine, Yonsei University College of Medicine, Seoul, Republic of Korea

2. Division of Cardiology, Department of Internal Medicine, Yonsei University College of Medicine, Seoul, Republic of Korea

Acute myocardial infarction (AMI) remains a major cause of mortality and morbidity worldwide. It is widely accepted that inflammation plays an important role in the pathogenesis of ST-segment elevation myocardial infarction (STEMI). The immature granulocyte is a practical marker of local and systemic inflammation. This study aimed to evaluate the association between the delta neutrophil index (DNI), which reflects immature granulocytes, and severity of STEMI, as well as to determine the significance of the DNI as a prognostic marker in patients with STEMI who underwent reperfusion. This retrospective, observational cohort study was conducted between January 1, 2011 and June 30, 2017 at a single tertiary academic hospital that attends to 85,000 patients in the emergency department annually In 2007, multidisciplinary critical pathway, known as FIRST, was implemented in our institution. Our critical pathway for STEMI management was designed to reduce unnecessary inhospital time delays and provide standard care. The present study included consecutive patients who were prospectively integrated into the FIRST critical pathway program, with those admitted with STEMI and who underwent primary percutaneous coronary intervention ( $\mathrm{pPCl}$ ) between January 1, 2011 and June 30, 2017 being analysed. A total of 842 patients were enrolled in this study. According to the multivariable Cox proportional hazard model, higher DNI ratios at time-l (within $2 \mathrm{~h}$ of $\mathrm{pPCl}$; hazard ratio $[\mathrm{HR}], 1.075 ; 95 \%$ confidence interval $[\mathrm{Cl}]$ $1.046-1.108 ; p<0.001)$ and time-24 $(24 \mathrm{~h}$ after admission; HR, 1.066; 95\% Cl: 1.045 1.086; $p<0.001$ ) were significant risk factors for 30-day mortality (Table 1).
Specifically, DNI values $>2.5 \%$ at time-I (HR, 13.643; 95\% Cl: 8.13-22.897; $\mathrm{p}<0.001)$ and $>2.9 \%$ at time-24 (HR, 12.752; 95\% Cl: 7.308-22.252; $p<0.001$ ) associated with an increased risk of 30-day mortality. Moreover, increased DNI values at times I and 24 were significantly associated with increased risks of heart failure and contrast-induced nephropathy in patients with acute STEMI (Figure 1). The integrated discrimination improvement (IDI) and continuous net reclassification index (NRI) were assessed to evaluate added predictive value. The addition of the DNI yielded significantly positive IDI for DNI values at times-I and time-24 and the continuous $\mathrm{NRI}$ was also positive. When comparing the C-statistics of the DNI to those of other markers, the statistics of the DNI at times I and time 24 were not inferior to those of creatine kinase-MB (CK-MB), Tn- $\mathrm{T}$, Creactive protein and NT pro BNP (Figure 2).

\begin{tabular}{lcl}
\hline \multicolumn{1}{c}{ Variable } & $\mathrm{HR}(95 \% \mathrm{CI})$ & $\mathrm{p}$-value \\
\hline Male Sex (vs Female) & $0.411(0.228-0.739)$ & $0.003^{*}$ \\
GRACE score (per 1 point) & $1.021(1.013-1.029)$ & $<0.001^{*}$ \\
LVEF (per 1\%) & $0.936(0.914-0.957)$ & $<0.001^{*}$ \\
NT-pro BNP (per 10^3 pg/mL) & $1.017(0.994-1.041)$ & 0.151 \\
Creatine kinase-MB (per 1 ng/mL) & $1.002(1.000-1.005)$ & 0.067 \\
Glucose (per 1 mg/dL) & $0.999(0.996-1.002)$ & 0.573 \\
Diabetes mellitus & $1.165(0.595-2.281)$ & 0.657 \\
Stroke & $2.113(0.946-4.720)$ & 0.068 \\
Malignancy & $2.415(1.003-5.818)$ & $0.049^{*}$ \\
Chronic kidney disease & $1.034(0.416-2.566)$ & 0.943 \\
Door-to-balloon time (per 1 min) & $1.010(1.003-1.017)$ & $0.006^{*}$ \\
Procedure time (per 1 min) & $1.008(0.996-1.021)$ & 0.211 \\
Multi-vessel disease & $0.900(0.482-1.678)$ & 0.74 \\
LM artery involvement & $3.028(1.409-6.506)$ & $0.005^{*}$ \\
DNI Time-I (per 1\%) & $1.076(1.046-1.108)$ & $<0.001^{*}$ \\
\hline
\end{tabular}

Table 1. Multivariable Cox regression analysis for predictors of 30day mortality
In patients with STEMI, the severity of systemic sterile inflammation triggered by the release of damage-associated molecular patterns from the necrotic myocardium is closely associated with clinical outcomes of acute MI. Although our study can't fully clarify the plausible mechanism of association of DNI with prognosis of STEMI, the possible explanations include. The rapid expansion of circulating neutrophils to compensate for a loss of active neutrophils due to the massive consumption and destruction of mature cells in severe inflammation is one likely mechanism. In addition, hemodynamic instability or persistently severe inflammation according to an increase in the severity of STEMI, may affect critical regulatory mechanisms for neutrophil release from the bone marrow. Based on these mechanisms the DNI after reperfusion may be a promising biomarker for predicting severity and mortality in the early stages of STEMI.

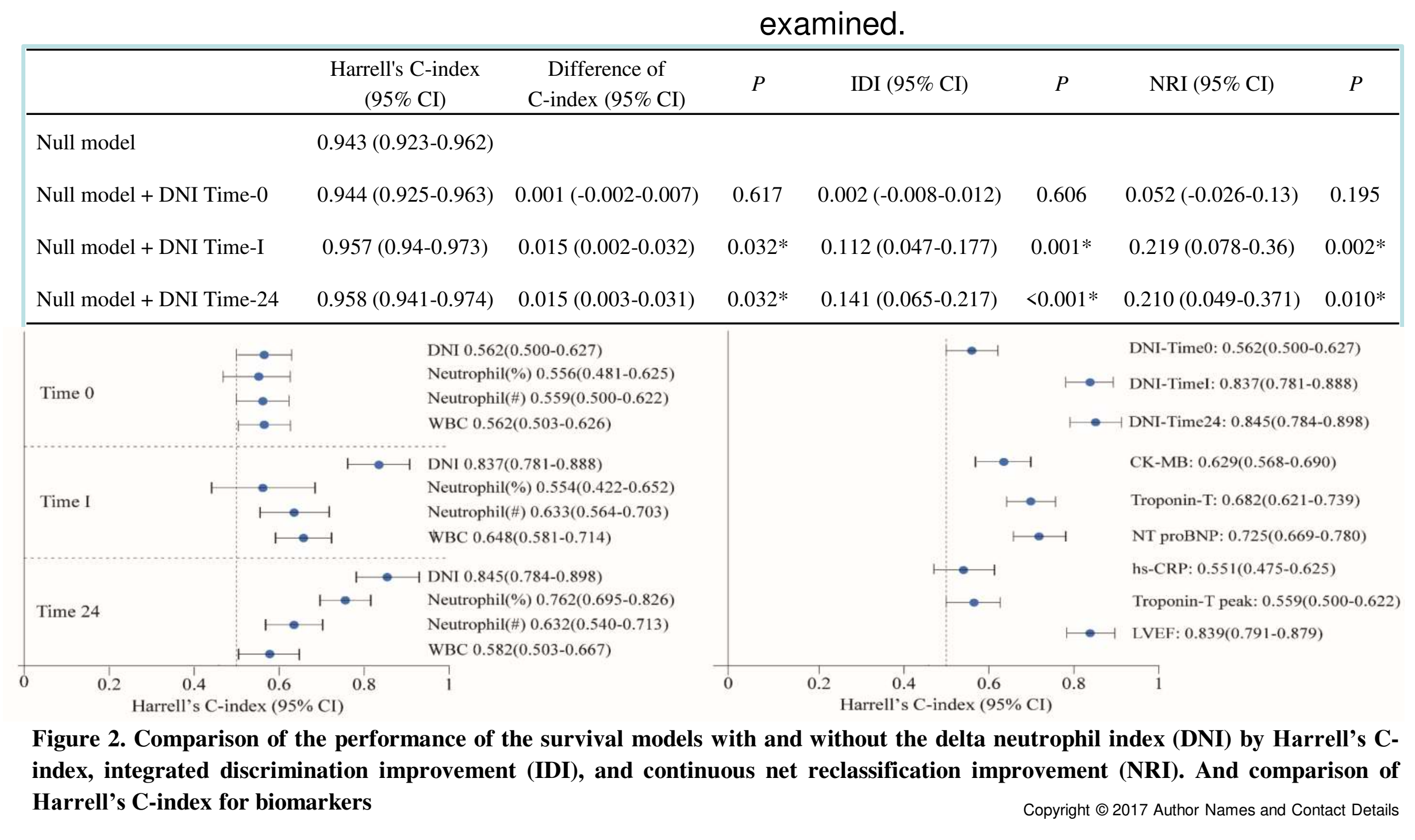

Figure 2. Comparison of the performance of the survival models with and without the delta neutrophil index (DNI) by Harrell's Cindex, integrated discrimination improvement (IDI), and continuous net reclassification improvement (NRI). And comparison of Harrell's C-index for biomarkers

Further large prospective studies are required to validate and compare the usefulness of DNI of severity as prognostic markers in patients with STEMI.

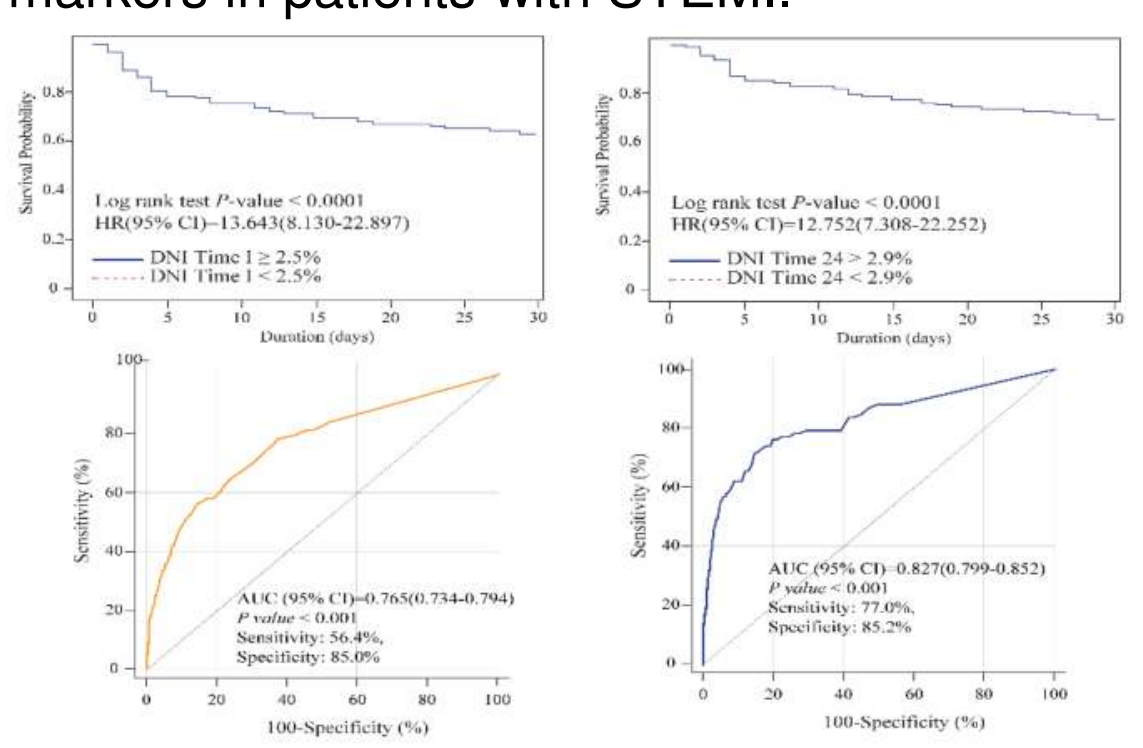

Figure 1. Higher DNI values within 2 hours post-pPCI was significantly associated with increased 30-das
risk and development of heart failure and CIN.

DNI values can be calculated without additional cost or time, and can be measured rapidly and simply. As higher DNI levels independently predict 30 -day mortality in patients with acute STEMI after $\mathrm{pPCl}$, the usefulness of this marker should be further examined. 